The Free Internet Journal

for Organic Chemistry
Paper

Arkivoc 2018, part ii, 72-80

\title{
Metal-free synthesis of $\alpha, \alpha$-difluorocyclopentanone derivatives via regioselective difluorocyclopropanation/VCP rearrangement of silyl dienol ethers
}

\author{
Ryo Takayama, Kohei Fuchibe, and Junji Ichikawa* \\ Division of Chemistry, Faculty of Pure and Applied Sciences, University of Tsukuba, \\ Tsukuba, Ibaraki 305-8571, Japan \\ Email: junji@chem.tsukuba.ac.jp
}

Dedicated to Professor Kenneth K. Laali in celebration of his 65th birthday

Received 08-01-2017

Accepted 08-20-2017

Published on line 10-29-2017

\section{Abstract}

Silyl dienol ethers underwent chemoselective difluorocyclopropanation under mild conditions with difluorocarbene, which was generated from trimethylsilyl 2,2-difluoro-2-(fluorosulfonyl)acetate (TFDA) using 1,8-bis(dimethylamino)naphthalene (proton sponge) as a catalyst. Successive vinylcyclopropane/cyclopentene (VCP) rearrangements readily proceeded to provide biologically promising 5,5-difluorocyclopent-1-en-1-yl silyl ethers in a one-pot operation without the use of metal catalysts.

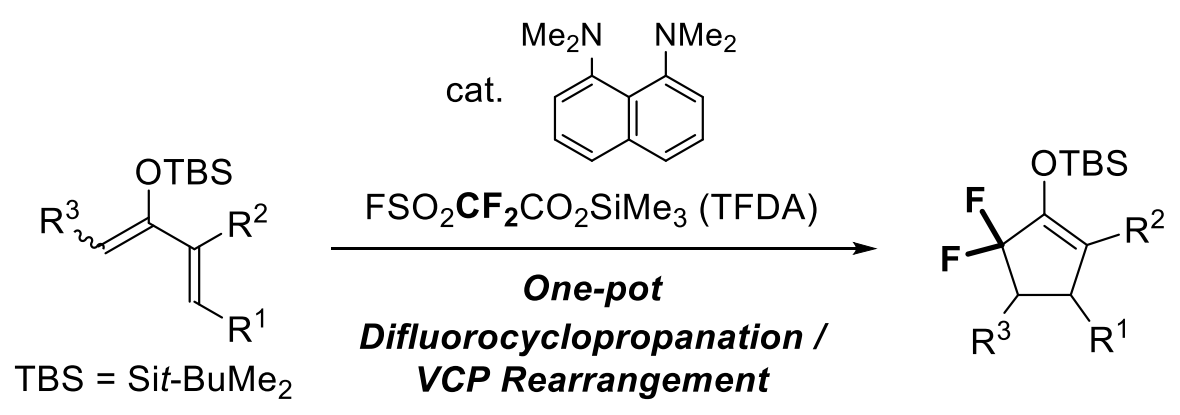

Keywords: Cyclopentanoid, difluorocarbene, difluorocyclopropanation, organocatalyst, vinylcyclopropane rearrangement 


\section{Introduction}

An important cyclopentanone framework is found in a variety of bioactive natural products and their synthetic intermediates. Their fluorinated counterparts are attracting much interest because of the biological effects of introducing fluorine. ${ }^{1,2}$ Till date, fluorinated cyclopentanone derivatives have been mostly synthesized by fluorination of cyclopentanones. ${ }^{3-6}$ However, these methods suffer from drawbacks relating to the requirement of time-consuming processes such as carbon-skeleton construction and fluorine installation as well as the use of expensive fluorinating reagents.

We have already reported the synthesis of $\alpha, \alpha$-difluorocyclopentanones based on the strategy of introducing difluoromethylene (Scheme 1). ${ }^{7}$ The reaction involves sequential (i) nickel-catalyzed chemoselective difluorocyclopropanation of silyl dienol ethers via a difluorocarbene complex and (ii) regioselective vinylcyclopropane/cyclopentene (VCP) rearrangement. ${ }^{8-10}$ This method is efficient because the introduction of fluorine substituents and the construction of the carbon skeleton are performed simultaneously. The five-membered cyclic silyl enol ethers thus obtained can be transformed to $\alpha, \alpha-$ difluorocyclopentanone derivatives.

Recently, we have also developed an organocatalytic method for the generation of difluorocarbene by the decomposition of trimethylsilyl 2,2-difluoro-2-(fluorosulfonyl)acetate (TFDA) ${ }^{11-13}$ (Scheme 2). ${ }^{14-17}$ The compound 1,8-bis(dimethylamino)naphthalene (proton sponge) was an efficient catalyst for the generation of difluorocarbene, with which silyl dienol ethers were chemoselectively transformed to the corresponding difluoro(vinyl)cyclopropanes, leading to the generation of $\alpha$-fluorocyclopentenones via a fluorine-directed and -activated Nazarov cyclization (Scheme 3, Path A). ${ }^{17}$ Thus, we envisioned that the organocatalytic generation of difluoro(vinyl)cyclopropanes followed by the aforementioned VCP rearrangement would result in a desirable metal-free process for the synthesis of $\alpha, \alpha$-difluorocyclopentanones (Scheme 3, Path B). ${ }^{18,} 19$

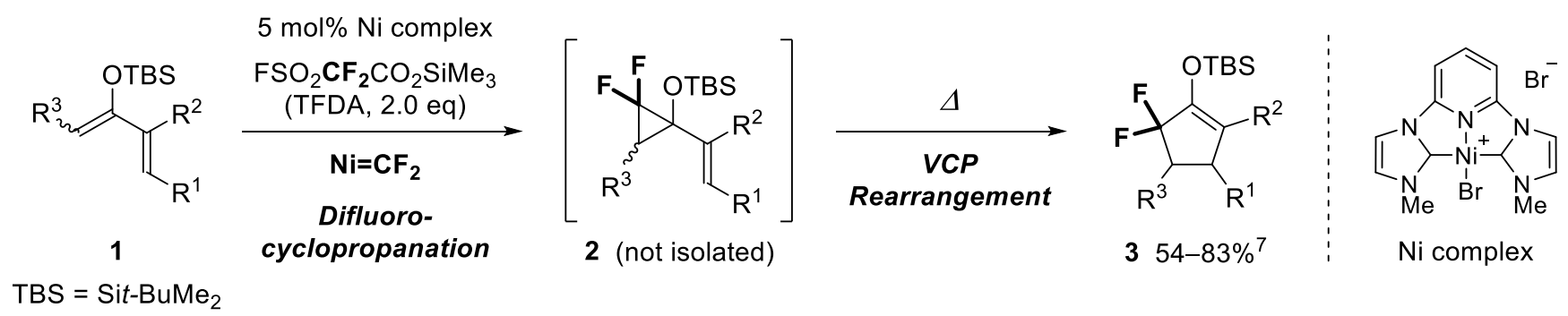

Scheme 1. Ni-catalyzed synthesis of 5,5-difluorocyclopent-1-en-1-yl silyl ethers.

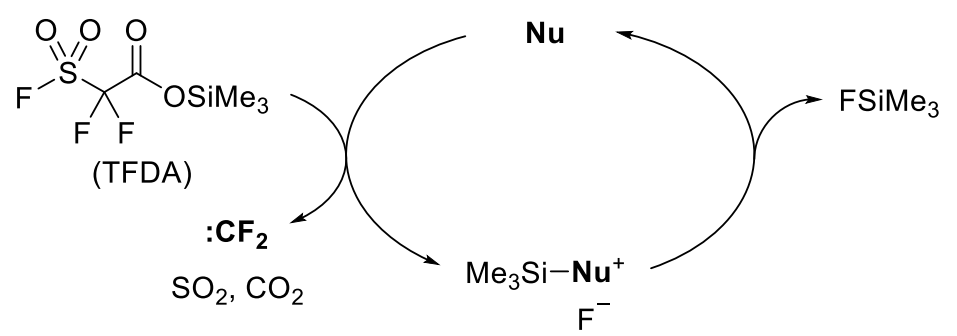

Nu (Organocatalyst)
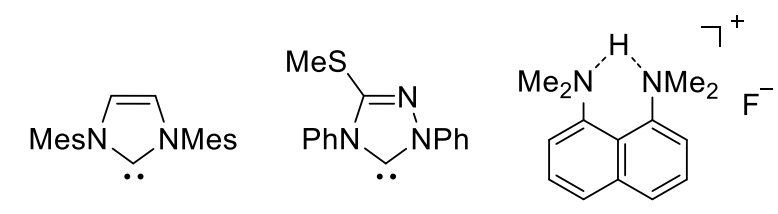

Scheme 2. Generation of difluorocarbene using organocatalysts. 


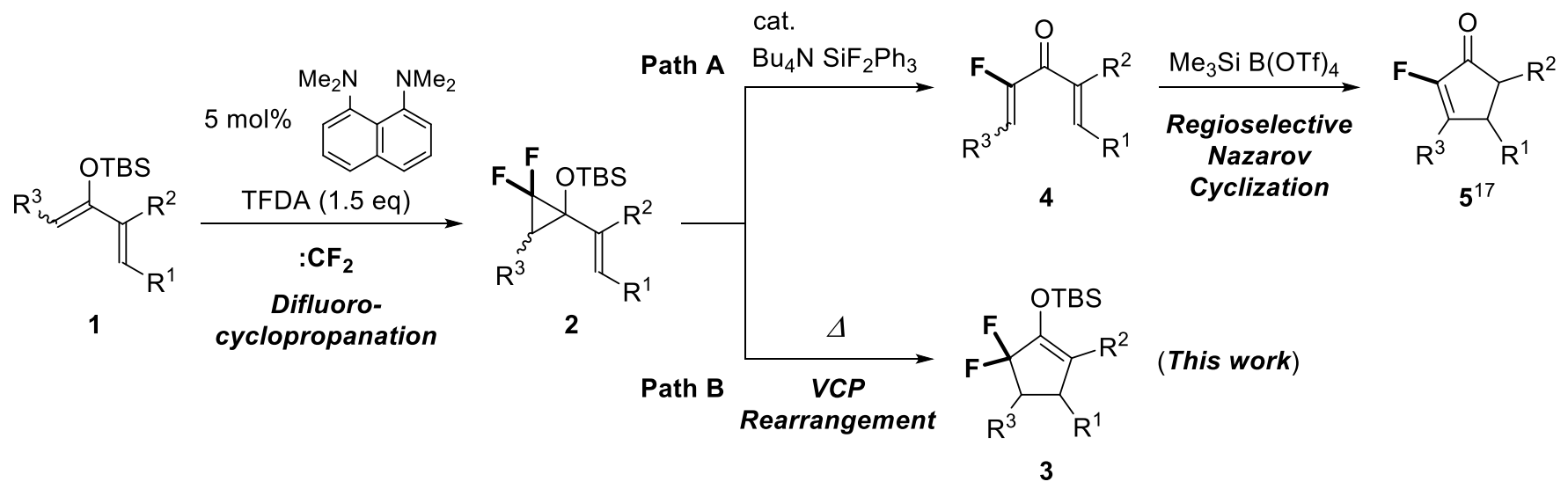

Scheme 3. Organocatalytic synthesis of fluorocyclopentanone derivatives.

\section{Results and Discussion}

VCP rearrangements of fluorine-free siloxyvinylcyclopropanes have been performed at high temperatures $\left(330-600{ }^{\circ} \mathrm{C}\right) \cdot{ }^{20-22}$ Fluorine substitution was reported to allow benign rearrangement conditions and render the $\mathrm{C}-\mathrm{C}$ bond cleavage regioselective. ${ }^{23-27}$ We first examined the temperature required for VCP rearrangement using isolated difluoro(vinyl)cyclopropane 2a. ${ }^{17}$ Cyclopropane 2a, prepared from silyl dienol ether $\mathbf{1 a}$ and difluorocarbene, was heated in $p$-xylene to give 5,5-difluorocyclopent-1-ene-1-yl silyl ether (cyclic silyl enol ether, $3 a$, Table 1). Thus, the rearrangement took place even at $80{ }^{\circ} \mathrm{C}$ (Table 1 , entries 1,2$)$, which was much lower than the temperature required for the VCP rearrangement of fluorine-free substrates. The rearrangement was found to proceed quickly $\left(30 \mathrm{~min}\right.$ ) at $140{ }^{\circ} \mathrm{C}$, affording $3 a$ in quantitative yield (Table 1 , entry 6).

Table 1. Optimization of reaction conditions for VCP rearrangement

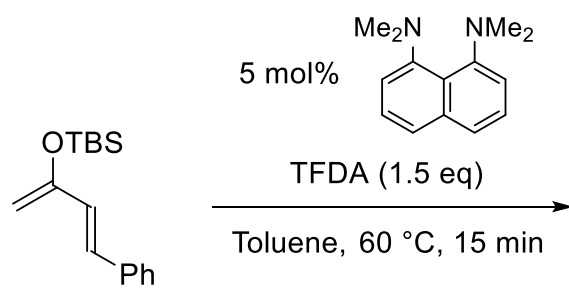

$1 a$

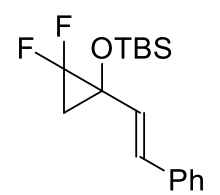

2a $76 \%$
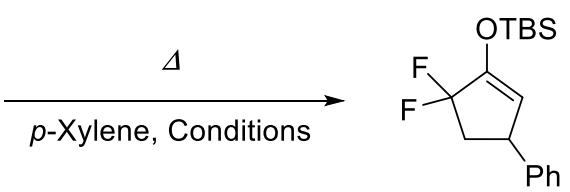

$3 a$

\begin{tabular}{lllll}
\hline Entry & Temp & Time & $3 \mathrm{a}^{\mathrm{a}}$ & Recovery of $\mathbf{2 \mathrm { a } ^ { \mathrm { a } }}$ \\
\hline 1 & $80^{\circ} \mathrm{C}$ & $1 \mathrm{~h}$ & $32 \%$ & $67 \%$ \\
2 & $80^{\circ} \mathrm{C}$ & $5 \mathrm{~h}$ & $80 \%$ & $14 \%$ \\
3 & $100^{\circ} \mathrm{C}$ & $1 \mathrm{~h}$ & $85 \%$ & $13 \%$ \\
4 & $100^{\circ} \mathrm{C}$ & $2 \mathrm{~h}$ & $96 \%$ & $\mathrm{nd}$ \\
5 & $120^{\circ} \mathrm{C}$ & $0.5 \mathrm{~h}$ & $92 \%$ & $4 \%$ \\
6 & $140^{\circ} \mathrm{C}$ & $0.5 \mathrm{~h}$ & Quant. & $\mathrm{nd}^{\mathrm{b}}$ \\
\hline
\end{tabular}

a ${ }^{19} \mathrm{~F}$ NMR yield based on an internal standard $\left(\mathrm{CF}_{3}\right)_{2} \mathrm{C}\left(\mathrm{C}_{6} \mathrm{H}_{4} \mathrm{CH}_{3}\right)_{2}$.

b not detected. 
Next, the desired metal-free, one-pot sequence of difluorocyclopropanation and VCP rearrangement was investigated (Table 2). Since, when a trace amount of acid remained in TFDA, decomposition of silyl dienol ethers 1 occurred predominantly during difluorocyclopropanation at room temperature, TFDA (1.5 equiv.) was added dropwise to the preheated solution of silyl dienol ether $1 \mathrm{a}$ and the proton sponge (5 mol\%) in $p$-xylene. The difluorocyclopropanation/VCP rearrangement proceeded efficiently at $140{ }^{\circ} \mathrm{C}$ in a one-pot operation to afford cyclic silyl enol ether $\mathbf{3 a}$ in $83 \%$ yield (Table 2 , entry 1 ). When silyl dienol ether $\mathbf{1} \mathbf{b}$ was used as the substrate, the reaction proceeded under the same conditions to afford $\mathbf{3 b}$ in $81 \%$ yield (Table 2, entry 2 ). The yield of $\mathbf{3 b}$ increased to $90 \%$ when difluorocyclopropanation was performed at $60^{\circ} \mathrm{C}^{17}$ followed by VCP rearrangement at $140{ }^{\circ} \mathrm{C}$ (Table 2 , entries 3,4$)$.

Table 2. Optimization of reaction conditions for sequential difluorocyclopropanation/VCP rearrangement

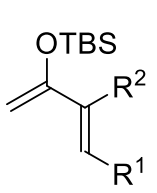

1

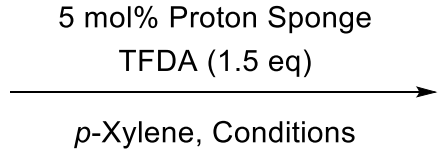

$p$-Xylene, Conditions

\begin{tabular}{cccccc}
\hline Entry & $\mathrm{R}^{1}$ & $\mathrm{R}^{2}$ & $\mathbf{1}$ & Conditions & $\mathbf{3}$ \\
\hline 1 & $\mathrm{Ph}$ & $\mathrm{H}$ & $\mathbf{1 a}$ & $140{ }^{\circ} \mathrm{C}, 30 \mathrm{~min}$ & $\mathbf{3 a ~} 83^{\mathrm{a}}$ \\
2 & $\mathrm{Ph}$ & $\mathrm{Me}$ & $\mathbf{1 b}$ & $140{ }^{\circ} \mathrm{C}, 30 \mathrm{~min}$ & $\mathbf{3 b} 81^{\mathrm{a}}$ \\
3 & $\mathrm{Ph}$ & $\mathrm{H}$ & $\mathbf{1 a}$ & $60^{\circ} \mathrm{C}, 15 \mathrm{~min} ;$ then $140{ }^{\circ} \mathrm{C}, 30 \mathrm{~min}$ & $\mathbf{3 a ~} 80^{\mathrm{b}}$ \\
4 & $\mathrm{Ph}$ & $\mathrm{Me}$ & $\mathbf{1 b}$ & $60^{\circ} \mathrm{C}, 15 \mathrm{~min}$; then $140{ }^{\circ} \mathrm{C}, 30 \mathrm{~min}$ & $\mathbf{3 b} 90^{\mathrm{b}}$ \\
\hline
\end{tabular}

${ }^{\text {a }}{ }^{19} \mathrm{~F}$ NMR yield based on an internal standard $\left(\mathrm{CF}_{3}\right)_{2} \mathrm{C}\left(\mathrm{C}_{6} \mathrm{H}_{4} \mathrm{CH}_{3}\right)_{2}$. ${ }^{\text {b }}$ Isolated yield.

Under optimized conditions, cyclic silyl enol ethers $\mathbf{3}$ with various substituents were synthesized (Table 3). Silyl dienol ethers $\mathbf{1} \mathbf{c}-\mathbf{f}$, bearing electron-donating or -withdrawing aryl or alkyl groups at the terminal position $\left(R^{1}\right)$, afforded the corresponding cyclic silyl enol ethers $\mathbf{3 c}-\mathbf{f}$ in $\mathbf{7 7 - 9 0 \% ~ y i e l d s . ~ S u b s t r a t e s ~} \mathbf{1 b}$ and $\mathbf{1 g}$, bearing a methyl or a bromo substituent at the internal position $\left(R^{2}\right)$, afforded $\mathbf{3 b}$ and $\mathbf{3 g}$ in $90 \%$ and $83 \%$ yields, respectively. When silyl dienol ether $\mathbf{1 h}$ with a cyclohexene ring was used as the substrate, $\mathbf{3 h}$ possessing a [4.3.0]bicyclononane structure was obtained in $66 \%$ yield.

Substrates $\mathbf{1} \mathbf{i}$ and $\mathbf{1} \mathbf{j}$, bearing an alkyl substituent (i.e., an isopropyl group and a butyl group, respectively) on the enol ether moiety $\left(R^{3}\right)$, also successfully participated in the reaction. ${ }^{28}$ Silyl dienol ethers $\mathbf{1} \mathbf{i}$ and $\mathbf{1} \mathbf{j}$ (with a $Z / E$ ratio of $92 / 8$ and $94 / 6$, respectively) underwent difluorocyclopropanation/VCP rearrangement, leading to the diastereoselective formation of $\mathbf{3} \mathbf{i}$ and $\mathbf{3} \mathbf{j}$ in $45 \%$ and $49 \%$ yields (with a trans/cis ratio of $>99 /<1$ and $>99 /<1$, respectively). These substrates exclusively provided more thermodynamically stable trans-isomers, probably because the ring closure avoided steric hindrance during the rearrangement. 
Table 3. Metal-free synthesis of cyclic silyl enol ethers 3a-ja

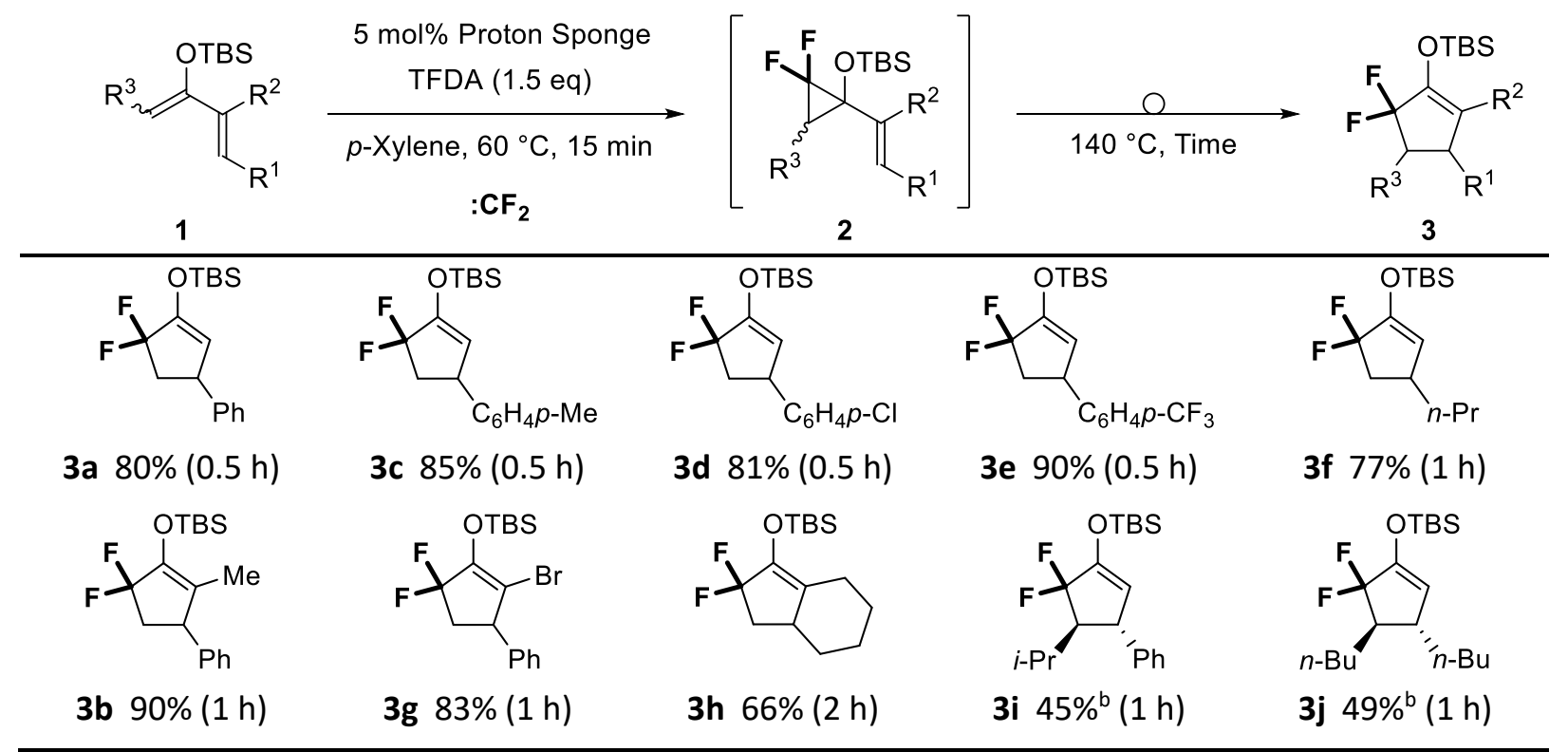

${ }^{a}$ Isolated yield (time). ${ }^{\mathrm{b}} \mathbf{3 i}$ and $\mathbf{3} \mathbf{j}$ were isolated as mixtures with siloxydienes. ${ }^{28}$

The metal-free protocol described here was advantageous with respect to regioselectivity in the VCP rearrangement step; this was exemplified by silyl dienol ether $\mathbf{1} \mathbf{g}$ (Scheme 4). Whereas $\mathbf{1 g}$ underwent metalfree difluorocyclopropanation/VCP rearrangement to give $\mathbf{3 g}$ as the sole five-membered product (Table 3 ), treatment of $\mathbf{1 g}$ with TFDA in the presence of a catalytic amount of a Ni complex afforded $\mathbf{3 g}$ in $72 \%$ yield along with the structural isomer 4,4-difluorocyclopent-1-en-1-yl silyl ether $6 \mathrm{~g}$ in $8 \%$ yield (Scheme 4)..$^{29}$ Thus, the advantage of the synthesis of 5,5-difluorocyclopent-1-en-1-yl silyl ethers 3 under metal-free conditions was demonstrated.

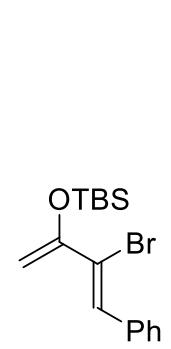

$1 \mathrm{~g}$
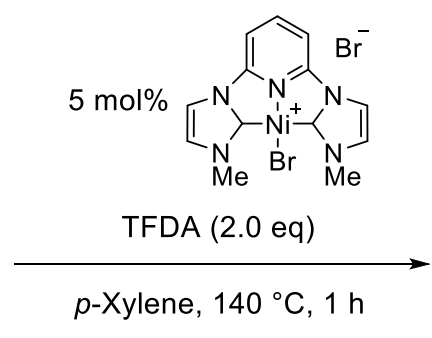

3 g $72 \%^{a}$

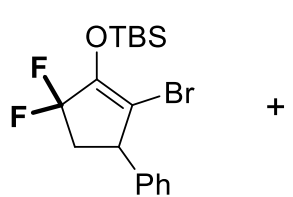

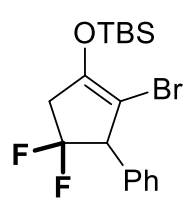

6 g $8 \%{ }^{a}$

Scheme 4. The disadvantage with the nickel-catalyzed protocol. a ${ }^{19} \mathrm{~F} \mathrm{NMR}$ yield based on an internal standard $\left(\mathrm{CF}_{3}\right)_{2} \mathrm{C}\left(\mathrm{C}_{6} \mathrm{H}_{4} \mathrm{CH}_{3}\right)_{2}$.

\section{Conclusions}

Metal-free difluorocyclopropanation/VCP rearrangement of silyl dienol ethers was successfully carried out with difluorocarbene generated from TFDA using a proton sponge as an organocatalyst. This resulted in complete regio- and stereoselective ring construction, leading to the efficient synthesis of biologically promising fluorinated cyclopentanones. 


\section{Experimental Section}

General. $p$-Xylene was stirred over anhydrous $\mathrm{CaCl}_{2}$, distilled after azeotoropic removal of water, and stored over molecular sieves 4A. Trimethylsilyl 2,2-difluoro-2-(fluorosulfonyl)acetate (TFDA) was prepared according to the literature. ${ }^{13}$ All of silyl dienol ethers (starting materials) $\mathbf{1 a}-\mathbf{j}$ were prepared according to the literature $^{7,17}$ and the spectral data were in good agreement with those in the literature.,17 1,8Bis(dimethylamino)naphthalene (proton sponge) and 1,1,1,3,3,3-hexafluoro-2,2-di(p-tolyl)propane (internal standard for ${ }^{19} \mathrm{~F}$ NMR) was purchased from Tokyo Chemical Industry Co., Ltd. Proton sponge was recrystalized from methanol/water.

Column chromatography and preparative thin layer chromatography (PTLC) were conducted on silica gel (Silica Gel 60 N, Kanto Chemical Co., Inc. for column chromatography and Wakogel B-5F, Wako Pure Chemical Industries, Ltd. for PTLC).

IR spectra were recorded on a Horiba FT-300S spectrometer by the attenuated total reflectance (ATR method). NMR spectra were recorded on a Bruker AVANCE 500 or a Jeol JNM ECS-400 spectrometer in $\mathrm{CDCl}_{3}$ at 500 or $400 \mathrm{MHz}\left({ }^{1} \mathrm{H} \mathrm{NMR}\right)$, at 126 or $100 \mathrm{MHz}\left({ }^{13} \mathrm{C} \mathrm{NMR}\right)$, and at 470 or $376 \mathrm{MHz}\left({ }^{19} \mathrm{~F} \mathrm{NMR}\right)$. Chemical shifts were given in ppm relative to internal $\mathrm{Me}_{4} \mathrm{Si}$ (for ${ }^{1} \mathrm{H} N M R: \delta=0.00$ ), $\mathrm{CDCl}_{3}$ (for ${ }^{13} \mathrm{C} \mathrm{NMR} \delta=77.0$ ), and $\mathrm{C}_{6} \mathrm{~F}_{6}\left(\right.$ for ${ }^{19} \mathrm{~F}$ NMR: $\delta=0.0$ ). High resolution mass spectroscopy (HRMS) was conducted with a Jeol JMS-T100GCV spectrometer (EI, TOF).

Typical procedure for the synthesis of 5,5-difluorocyclopent-1-en-1-yl silyl ethers (3). Synthesis of 3a is described as a typical procedure. The mixture of proton sponge $(2.3 \mathrm{mg}, 0.011 \mathrm{mmol}), 1,1,1,3,3,3$-hexafluoro2,2-di(p-tolyl)propane $(6.2 \mathrm{mg}, 0.019 \mathrm{mmol})$, and silyl dienol ether 1a $(52 \mathrm{mg}, 0.20 \mathrm{mmol})$ in $p$-xylene $(2 \mathrm{~mL})$ was heated to $60{ }^{\circ} \mathrm{C}$ and TFDA ( $60 \mu \mathrm{L}, 0.30 \mathrm{mmol}$ ) was added dropwise over $5 \mathrm{~min}$. The resulting mixture was stirred at $60{ }^{\circ} \mathrm{C}$ for $15 \mathrm{~min}$. The reaction mixture was heated at $140{ }^{\circ} \mathrm{C}$ for $30 \mathrm{~min}$ and then cooled to room temperature. The mixture was diluted with hexane $(2 \mathrm{~mL})$ and a saturated aqueous solution $(10 \mathrm{~mL})$ of sodium hydrogen carbonate was added. Organic materials were extracted with hexane three times. The combined extracts were dried over anhydrous sodium sulfate. The sulfate was removed by filtration and the filtrate was concentrated under reduced pressure. The residue was purified by column chromatography on silica gel (hexane) to afford 5,5-difluorocyclopent-1-en-1-yl silyl ether $3 a$ as a yellow liquid (50 $\mathrm{mg}, 80 \%$ yield).

Spectral data of $\mathbf{3 a}, \mathbf{3 b}, \mathbf{3 c}, \mathbf{3 d}, \mathbf{3 f}, \mathbf{3 g}$, and $\mathbf{3 h}$ showed complete agreement with those in our previous paper. ${ }^{7}$ 1-[tert-Butyl(dimethyl)silyloxy]-5,5-difluoro-3-[4-(trifluoromethyl)phenyl]cyclopent-1-en (3e). ${ }^{1} \mathrm{H}$ NMR (400 $\mathrm{MHz}_{1} \mathrm{CDCl}_{3}$ ): $\delta 0.23(\mathrm{~s}, 3 \mathrm{H}), 0.24(\mathrm{~s}, 3 \mathrm{H}), 0.98(\mathrm{~s}, 9 \mathrm{H}), 2.14$ (dddd, J 17.2, 16.0, 11.6, $4.0 \mathrm{~Hz}, 1 \mathrm{H}$ ), 2.84 (dddd, J 16.0, 16.0, 8.4, 8.4 Hz, 1H), $3.86-3.93(\mathrm{~m}, 1 \mathrm{H}), 5.16(\mathrm{~d}, J 2.0 \mathrm{~Hz}, 1 \mathrm{H}), 7.31(\mathrm{~d}, J 8.0 \mathrm{~Hz}, 2 \mathrm{H}), 7.57(\mathrm{~d}, J 8.0 \mathrm{~Hz}, 2 \mathrm{H})$. ${ }^{13} \mathrm{C}$ NMR $\left(100 \mathrm{MHz}, \mathrm{CDCl}_{3}\right): \delta-4.8,-4.8,18.2,25.5,40.5,41.6(\mathrm{dd}, J 25,25 \mathrm{~Hz}), 114.3(\mathrm{dd}, J \mathrm{~J}, 7 \mathrm{~Hz}), 124.1$ (q, J $270 \mathrm{~Hz}$ ), 125.7, 125.8, 126.8 (dd, J 243, $243 \mathrm{~Hz}$ ), 127.4, 148.1 (d, J $5 \mathrm{~Hz}$ ), 149.3 (dd, J 25, $25 \mathrm{~Hz}$ ). ${ }^{19} \mathrm{~F} \mathrm{NMR} \mathrm{(376}$ $\mathrm{MHz}_{\mathrm{CDCl}}$ ): $\delta 99.3$ (s, 1F), 69.3 (dddd, J 8, 249, 17, 11, $\left.8 \mathrm{~Hz}, 1 \mathrm{~F}\right), 64.5$ (dddd, J 249, 16, 12, $3 \mathrm{~Hz}, 1 \mathrm{~F}$ ). IR (neat): v 2933, 2862, 1655, 1323, 1167, 1068, $835 \mathrm{~cm}^{-1}$. HRMS (70 eV, El): $\mathrm{m} / z$ calcd. for $\mathrm{C}_{14} \mathrm{H}_{14} \mathrm{~F}_{5} \mathrm{OSi}[\mathrm{M}-t-\mathrm{Bu}]^{+}$: 321.0734; Found: 321.0732.

1-[tert-Butyl(dimethyl)silyloxy]-5,5-difluoro-4-isopropyl-3-phenylcyclopent-1-ene (3i). ${ }^{1} \mathrm{H} \mathrm{NMR}(400 \mathrm{MHz}$, $\left.\mathrm{CDCl}_{3}\right): \delta 0.20(\mathrm{~s}, 3 \mathrm{H}), 0.21(\mathrm{~s}, 3 \mathrm{H}), 0.86(\mathrm{~d}, J 6.4 \mathrm{~Hz}, 3 \mathrm{H}), 0.97(\mathrm{~s}, 9 \mathrm{H}), 1.04(\mathrm{~d}, J 6.4 \mathrm{~Hz}, 3 \mathrm{H}), 2.02-2.20(\mathrm{~m}, 2 \mathrm{H})$, 3.50-3.58 (m, 1H), $5.04(\mathrm{dd}, J$ J.8, $1.8 \mathrm{~Hz}, 1 \mathrm{H}), 7.21(\mathrm{~d}, J 7.6 \mathrm{~Hz}, 2 \mathrm{H}), 7.22(\mathrm{t}, J 7.6 \mathrm{~Hz}, 1 \mathrm{H}), 7.30(\mathrm{dd}, J 7.6,7.6 \mathrm{~Hz}$, 2H). ${ }^{13} \mathrm{C}$ NMR $\left(100 \mathrm{MHz}, \mathrm{CDCl}_{3}\right): \delta-4.9,-4.8,18.2,20.8,21.5,25.5,27.9(\mathrm{~d}, J 5 \mathrm{~Hz}), 46.3(\mathrm{~d}, J 7 \mathrm{~Hz}), 58.5(\mathrm{dd}, J$ 19, $19 \mathrm{~Hz}$ ), 115.9 (dd, J 9, $9 \mathrm{~Hz}), 126.7,127.4$ (dd, J 247, $247 \mathrm{~Hz}$ ), 127.7, 128.6, 144.4 (d, J $5 \mathrm{~Hz}$ ), 147.6 (dd, J 23, $23 \mathrm{~Hz}$ ). ${ }^{19} \mathrm{~F} \mathrm{NMR}\left(376 \mathrm{MHz}, \mathrm{CDCl}_{3}\right.$ ): $\delta 52.1$ (ddd, J 251, 13, $\left.4 \mathrm{~Hz}, 1 \mathrm{~F}\right), 70.5$ (ddd, J 251, 19, $10 \mathrm{~Hz}, 1 \mathrm{~F}$ ). 
IR (neat): v 2958, 2931, 2860, 1660, 1365, 1188, 1011, $839 \mathrm{~cm}^{-1}$. HRMS (70 eV, El): $\mathrm{m} / \mathrm{z}$ calcd. for $\mathrm{C}_{16} \mathrm{H}_{21} \mathrm{~F}_{2} \mathrm{OSi}$ $[\mathrm{M}-t-\mathrm{Bu}]^{+}:$295.1330; Found: 295.1329.

3,4-Dibutyl-1-[tert-butyl(dimethyl)silyloxy]-5,5-difluorocyclopent-1-ene (3j). ${ }^{1} \mathrm{H} \mathrm{NMR}\left(400 \mathrm{MHz}^{\mathrm{CDCl}} 3\right.$ ): $\delta$ $0.179(\mathrm{~s}, 3 \mathrm{H}), 0.183(\mathrm{~s}, 3 \mathrm{H}), 0.87-0.93(\mathrm{~m}, 6 \mathrm{H}), 0.95(\mathrm{~s}, 9 \mathrm{H}), 1.21-1.53(\mathrm{~m}, 12 \mathrm{H}), 1.59-1.70(\mathrm{~m}, 1 \mathrm{H}), 1.80-1.93$ (m, 1H), 5.09 (br s, 1H). $\left.{ }^{13} \mathrm{C} \mathrm{NMR} \mathrm{(100} \mathrm{MHz,} \mathrm{CDCl}\right): \delta-4.91,-4.85,13.9,14.0,18.2,22.9$ (d, J $\left.5 \mathrm{~Hz}\right), 25.5,28.0$, 28.1, 29.5, 30.0, 35.0 (d, J 5 Hz), 42.2 (d, J 7 Hz), 49.3 (dd, J 22, 22 Hz), 115.2 (dd, J 8, 8 Hz), 127.2 (dd, J 245, $245 \mathrm{~Hz}$ ), 147.0 (dd, J 25, $25 \mathrm{~Hz}$ ). ${ }^{19} \mathrm{~F} \mathrm{NMR}\left(376 \mathrm{MHz}, \mathrm{CDCl}_{3}\right): \delta 53.5$ (ddd, J 249, 11, $\left.3 \mathrm{~Hz}, 1 \mathrm{~F}\right), 69.8$ (ddd, J 249, 20, $11 \mathrm{~Hz}, 1 \mathrm{~F})$. IR (neat): v 2958, 2929, 2860, 1660, 1363, 1186, 1012, $839 \mathrm{~cm}^{-1}$. HRMS (70 eV, El): $\mathrm{m} / \mathrm{z}$ calcd. for $\mathrm{C}_{15} \mathrm{H}_{27} \mathrm{~F}_{2} \mathrm{OSi}[\mathrm{M}-t-\mathrm{Bu}]^{+}:$289.1799; Found: 289.1799.

2-Bromo-1-[tert-butyl(dimethyl)silyloxy]-4,4-difluoro-3-phenylcyclopent-1-ene (6g). ${ }^{1} \mathrm{H} \mathrm{NMR}(500 \mathrm{MHz}$, $\mathrm{CDCl}_{3}$ ): $\delta 0.280(\mathrm{~s}, 3 \mathrm{H}), 0.283(\mathrm{~s}, 3 \mathrm{H}), 1.01(\mathrm{~s}, 9 \mathrm{H}), 2.85$ (ddd, J 16.5, 16.5, $\left.7.5 \mathrm{~Hz}, 1 \mathrm{H}\right), 2.88-2.97(\mathrm{~m}, 1 \mathrm{H}), 4.20$ (dd, J 20.0, $4.5 \mathrm{~Hz}, 1 \mathrm{H}), 7.19-7.20(\mathrm{~m}, 2 \mathrm{H}), 7.32-7.38(\mathrm{~m}, 3 \mathrm{H}) .{ }^{13} \mathrm{C} \mathrm{NMR}\left(126 \mathrm{MHz}, \mathrm{CDCl}_{3}\right): \delta-4.0,-3.9,18.1$, 25.5, 43.3 (dd, J 28, 28 Hz), 60.8 (dd, J 28, 23 Hz), 95.6 (d, J 3 Hz), 124.4 (dd, J 259, 254 Hz), 128.1, 128.5, 129.1, 133.8 (dd, J 4, $4 \mathrm{~Hz}$ ), 147.2 (dd, J 7, $4 \mathrm{~Hz}$ ). ${ }^{19} \mathrm{~F} \mathrm{NMR} \mathrm{(470} \mathrm{MHz,} \mathrm{CDCl} 3$ ): $\delta 65.5$ (dddd, J 227, 14, 8, $5 \mathrm{~Hz}, 1 \mathrm{~F}$ ), 75.2 (dddd, J 227, 20, 18, $15 \mathrm{~Hz}, 1 \mathrm{~F}$ ). IR (neat): v 2925, 2856, 1662, 1327, 1255, 1117, 912, $839 \mathrm{~cm}^{-1}$. HRMS (70 eV, EI): $m / z$ calcd. for $\mathrm{C}_{17} \mathrm{H}_{23} \mathrm{BrF}_{2} \mathrm{OSi}[\mathrm{M}]^{+}: 388.0670$; Found: 388.0669.

3-[tert-Butyl(dimethyl)silyloxy]-4,4-difluoro-6-methyl-1-phenylhepta-2,5-diene (7i). ${ }^{1} \mathrm{H} N M R(400 \mathrm{MHz}$, $\left.\mathrm{CDCl}_{3}\right): \delta 0.14(\mathrm{~s}, 6 \mathrm{H}), 0.91(\mathrm{~s}, 9 \mathrm{H}), 1.79(\mathrm{td}, J 3.2,1.4 \mathrm{~Hz}, 3 \mathrm{H}), 1.84(\mathrm{td}, J 2.8,1.4 \mathrm{~Hz}, 3 \mathrm{H}), 3.50-3.58(\mathrm{~m}, 2 \mathrm{H})$, $5.03(\mathrm{t}, J 1.8 \mathrm{~Hz}, 1 \mathrm{H}), 5.50$ (tqq, J 13.3, 1.4, $1.4 \mathrm{~Hz}, 1 \mathrm{H}), 7.19-7.43(\mathrm{~m}, 5 \mathrm{H}) .{ }^{13} \mathrm{C} \mathrm{NMR}\left(126 \mathrm{MHz}, \mathrm{CDCl}_{3}\right): \delta-4.7$, 18.0, 25.5, 26.5, 29.7, 31.6 (t, J $4 \mathrm{~Hz}), 111.3,118.0$ (t, J $239 \mathrm{~Hz}), 120.7$ (t, J $28 \mathrm{~Hz}), 125.9,128.2,128.4,141.1$, 142.7 (t, J $7 \mathrm{~Hz}$ ), 146.0 (t, J $30 \mathrm{~Hz}$ ). ${ }^{19} \mathrm{~F} \mathrm{NMR}\left(376 \mathrm{MHz}, \mathrm{CDCl}_{3}\right.$ ): $\delta 53.5$ (br d, J $13 \mathrm{~Hz}$ ). HRMS (70 eV, El): m/z calcd. for $\mathrm{C}_{16} \mathrm{H}_{21} \mathrm{~F}_{2} \mathrm{OSi}[\mathrm{M}-t-\mathrm{Bu}]^{+}$: 295.1330; Found: 295.1330 .

7-[tert-Butyl(dimethyl)silyloxy]-6,6-difluorotrideca-4,7-diene (7j). ${ }^{1} \mathrm{H} \mathrm{NMR}\left(400 \mathrm{MHz}, \mathrm{CDCl}_{3}\right): \delta 0.14(\mathrm{~s}, 6 \mathrm{H})$, $0.91(\mathrm{~s}, 9 \mathrm{H}), 0.95-1.01(\mathrm{~m}, 6 \mathrm{H}), 1.21-1.53(\mathrm{~m}, 8 \mathrm{H}), 2.06-2.17(\mathrm{~m}, 4 \mathrm{H}), 4.93(\mathrm{t}, \mathrm{J} 8.0 \mathrm{~Hz}, 1 \mathrm{H}), 5.60-5.71(\mathrm{~m}, 1 \mathrm{H})$, 6.10 (dtt, J 15.6, 9.6, $2.8 \mathrm{~Hz}, 1 \mathrm{H}) .{ }^{13} \mathrm{C} \mathrm{NMR}\left(100 \mathrm{MHz}, \mathrm{CDCl}_{3}\right): \delta-4.7,13.6,14.0,18.1,21.7,22.5,25.2,25.6$, 30.1, 31.4, 33.8, 113.9, 117.7 (t, J $240 \mathrm{~Hz}), 124.6$ (t, J $28 \mathrm{~Hz}), 136.1$ (t, J $10 \mathrm{~Hz}), 143.9$ (t, J $30 \mathrm{~Hz}) .{ }^{19} \mathrm{~F} \mathrm{NMR}(376$ $\mathrm{MHz}, \mathrm{CDCl}_{3}$ ): $\delta 67.8$ (ddd, J 10, 6, $3 \mathrm{~Hz}$ ). HRMS (70 eV, El): $\mathrm{m} / z$ calcd. for $\mathrm{C}_{15} \mathrm{H}_{27} \mathrm{~F}_{2} \mathrm{OSi}[\mathrm{M}-t-\mathrm{Bu}]^{+}: 289.1799$; Found: 289.1798.

\section{Acknowledgements}

This research is supported by JSPS KAKENHI Grant Number JP16K13943 (J.I.), JSPS KAKENHI Grant Number JP16H01002 (J.I.), JSPS KAKENHI Grant Number JP15K05414 (K.F.). Shin-Etsu Chemical Co., Ltd., is gratefully acknowledged for the generous gift of TBSCl.

\section{Supplementary Material}

${ }^{1} \mathrm{H},{ }^{13} \mathrm{C}$, and ${ }^{19} \mathrm{~F} N M R$ data of new compounds $(\mathbf{3 e}, \mathbf{3 i}, \mathbf{3} \mathbf{j}, \mathbf{6} \mathbf{g}, \mathbf{7} \mathbf{i}$, and $\mathbf{7 j})$ are shown.

\section{References and Notes}

1. Kirsch, P. Modern Fluoroorganic Chemistry: Synthesis, Reactivity, Applications; Wiley-VCH: Weinheim, 2004. 
2. Uneyama, K. Organofluorine Chemistry; Blackwell: Oxford, 2006.

3. Baudoux, J.; Cahard, D. Org. React. 2007, 69, 347-669.

4. Nakano, T.; Makino, M.; Morizawa, Y.; Matsumura, Y. Angew. Chem., Int. Ed. Engl. 1996, 35, $1019-1021$. https://doi.org/10.1002/anie.199610191

5. Pravst, I.; Zupan, M.; Stavber, S. Synthesis 2005, 2005, 3140-3146.

6. Meegalla, S. K.; Doller, D.; Liu, R.; Sha, D.; Lee, Y.; Soll, R. M.; Wisnewski, N.; Silver, G. M.; Dhanoa, D. Bioorg. Med. Chem. Lett. 2006, 16, 1702-1706.

https://doi.org/10.1016/j.bmcl.2005.12.012

7. Aono, T.; Sasagawa, H.; Fuchibe, K.; Ichikawa, J. Org. Lett. 2015, 17, 5736-5739.

https://doi.org/10.1021/acs.orglett.5b02726

8. Hudlicky, T.; Kutchan, T. M.; Naqvi, S. M. Org. React. 1985, 33, 247-335.

9. Wong, H. N. C.; Hon, M. Y.; Tse, C. W.; Yip, Y. C.; Tanko, J.; Hudlicky, T. Chem. Rev. 1989, 89, $165-198$. https://doi.org/10.1021/cr00091a005

10. Baldwin, J. E. Chem. Rev. 2003, 103, 1197-1212.

https://doi.org/10.1021/cr010020z

11. Terjeson, R. J.; Mohtasham, J.; Peyton, D. H.; Gard, G. L.; J. Fluorine Chem. 1989, 42, 187-200.

https://doi.org/10.1016/S0022-1139(00)82749-9

12. Tian, F.; Kruger, V.; Bautista, O.; Duan, J.-X.; Li, A.-R.; Dolbier, W. R., Jr.; Chen, Q.-Y. Org. Lett. 2000, 2, 563-564.

https://doi.org/10.1021/ol0055622

13. Dolbier, W. R., Jr.; Tian, F.; Duan, J.-X.; Li, A.-R.; Ait-Mohand, S.; Bautista, O.; Buathong, S.; Baker, J. M.; Crawford, J.; Anselme, P.; Cai, X. H.; Modzelewska, A.; Koroniak, H.; Battiste, M. A.; Chen, Q.-Y. J. Fluorine Chem. 2004, 125, 459-469.

https://doi.org/10.1016/i.jfluchem.2003.12.002

14. Fuchibe, K.; Koseki, Y.; Sasagawa, H.; Ichikawa, J. Chem. Lett. 2011, 40, 1189-1191.

https://doi.org/10.1246/cl.2011.1189

15. Fuchibe, K.; Koseki, Y.; Aono, T.; Sasagawa, H.; Ichikawa, J. J. Fluorine Chem. 2012, 133, 52-60.

https://doi.org/10.1016/j.jfluchem.2011.09.012

16. Fuchibe, K.; Bando, M.; Takayama, R.; Ichikawa, J. J. Fluorine Chem. 2015, 171, 133-138.

https://doi.org/10.1016/j.jfluchem.2014.08.013

17. Fuchibe, K.; Takayama, R.; Yokoyama, T.; Ichikawa, J. Chem. Eur. J. 2017, 23, 2831-2838.

https://doi.org/10.1002/chem.201604578

18. Independently, ammonium bromide-catalyzed synthesis of $\alpha, \alpha$-difluorocyclopentanones with (bromodiflioromethyl)trimethylsilane was conducted quite recently. See: ref. 19

19. Chang, J.; Xu, C.; Gao, J.; Gao, F.; Zhu, D.; Wang, M. Org. Lett. 2017, 19, 1850-1853. https://doi.org/10.1021/acs.orglett.7b00611

20. Trost, B. M.; Bogdanowicz, M. J. J. Am. Chem. Soc. 1973, 95, 289-290. https://doi.org/10.1021/ja00782a077

21. Girard, C.; Amice, P.; Barnier, J. P.; Conia, J. M. Tetrahedron Lett. 1974, 15, 3329-3332. https://doi.org/10.1016/S0040-4039(01)91899-4

22. Rousseau, G.; Slougui, N. Tetrahedron Lett. 1983, 24, 1251-1254.

https://doi.org/10.1016/S0040-4039(00)81627-5

23. Dolbier, W. R., Jr.; Sellers, S. F. J. Am. Chem. Soc. 1982, 104, 2494-2497.

https://doi.org/10.1021/ja00373a026 
24. Dolbier, W. R., Jr.; Sellers, S. F. J. Org. Chem. 1982, 47, 1-4.

https://doi.org/10.1021/jo00340a001

25. Mitsch, R. A.; Neuvar, E. W. J. Phys. Chem. 1966, 70, 546-553.

https://doi.org/10.1021/j100874a039

26. Smart, B. E.; Krusic, P. J.; Roe, D. C.; Yang, Z.-Y. J. Fluorine Chem. 2002, 117, 199-205. https://doi.org/10.1016/S0022-1139(02)00187-2

27. Orr, D.; Percy, J. M.; Tuttle, T.; Kennedy, A. R.; Harrison, Z. A. Chem. Eur. J. 2014, 20, 14305-14316. https://doi.org/10.1002/chem.201403737

28. When the substrates $\mathbf{1} \mathbf{i}$ and $\mathbf{1} \mathbf{j}$ with an isopropyl group and a butyl group as $\mathrm{R}^{3}$ were employed, the corresponding products $\mathbf{3 i}$ and $\mathbf{3} \mathbf{j}$ were obtained along with siloxydienes $\mathbf{7} \mathbf{i}$ and $\mathbf{7 j}$ as $\mathbf{1 , 5}$-hydrogen shift products $(3 \mathbf{i}: 7 \mathbf{i}=86: 14, \mathbf{3 j}: 7 \mathbf{j}=81: 19)$ in $52 \%$ and $60 \%$ yields, respectively.

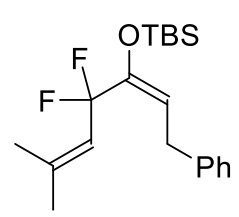

$7 \mathbf{i}$

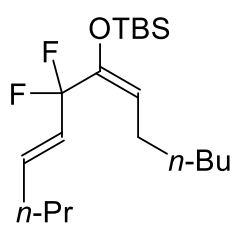

7j

29. Although the formation mechanism of $6 \mathrm{~g}$ is uncertain, it could be generated by a formal [4+1] cycloaddition ${ }^{30}$ or an oxidative addition of the $\mathrm{C}-\mathrm{C}$ bond whose carbons are substituted by fluorine and siloxy group in cyclopropane $\mathbf{2 g}$ to a $\mathrm{Ni}(0)$ complex followed by ring expansion and reductive elimination.

30. Fuchibe, K.; Aono, T.; Hu, J.; Ichikawa, J. Org. Lett. 2016, 18, 4502-4505. https://doi.org/10.1021/acs.orglett.6b02085 